\title{
The ying and yang of fever in rheumatic disease
}

\author{
Authors: James Galloway ${ }^{A}$ and Andrew P Cope ${ }^{B}$
}

Fevers are relatively common in rheumatic disease, largely due to the fact that the inflammatory process is driven by inflammatory mediators that function as endogenous pyrogens. Since the immune system's sensors cannot accurately distinguish between endogenous and exogenous (pathogen-derived) pyrogens a major challenge for physicians and rheumatologists has been to decipher patterns of clinical signs and symptoms to inform clinical decision making. Here we describe some of the common pitfalls and clinical challenges, and highlight the importance of a systematic approach to investigating the rheumatic disease patient presenting with fever.

\section{Introduction}

The onset of fever reflects an adaptive response of organisms to exogenous and endogenous pyrogens. This complex physiological reaction, widespread in the animal kingdom, is manifest by expression of pyrogenic cytokines, acute phase reactants and activation of a myriad of immune, neurological and endocrine systems, serving to promote protective host responses. In the clinical setting, fever rarely exists as an isolated clinical entity, being commonly associated with somnolence, anorexia, malaise and fatigue. These signatures are well recognised by physicians caring for patients with inflammatory disease. Nonetheless, a major challenge exists in identifying whether the primary pyrogenic stimulus is exogenous (eg microbe or toxin) or driven by aseptic, endogenous factors. Despite intense research, there exist no clear cellular and molecular signatures that discriminate between an acute inflammatory flare and an acute infectious episode in the context of systemic chronic inflammatory disease because induction of immune and inflammatory mediators is very similar. Thus, for the time being, clinical acumen, through recognition of patterns of symptoms and signs, remains an essential decision-making tool.

\section{Fevers in the context of rheumatic disease}

Descriptions of fever in the context of rheumatic diseases date back to Hippocrates, with early descriptions of rheumatic

Authors: Aclinical lecturer and honorary consultant in rheumatology, Academic Department of Rheumatology, King's College London, London, UK; ' BArthritis Research UK professor of rheumatology, Academic Department of Rheumatology, Centre for Molecular and Cellular Biology of Inflammation, King's College London, London, UK fever. ${ }^{1}$ The modern rheumatologist is frequently faced with questions relating to fever, often with respect to investigation of fever of unknown origin. Recent studies estimate approximately $20 \%$ of cases of fever of unknown origin have an underlying inflammatory cause, commonly adult-onset Still's disease (AOSD) and large vessel vasculitis. ${ }^{2}$ At other times, rheumatologists are involved in the assessment of fever attributable to an infection complicating immune suppression. For the purpose of this review, we will consider the relationship between fever and the major classes of inflammatory rheumatic diseases in turn. In general we will refer to fever as a body temperature above $38^{\circ} \mathrm{C}$ (accepting definitions of the upper limit of normal range from $37.5-38 \cdot 3^{\circ} \mathrm{C}$ ). Appreciating which rheumatic diseases are associated with fever is essential for deciding how far to pursue alternative causes.

\section{Monoarthritis and fever}

\section{Crystal arthritis}

Gout is the most prevalent form of crystal arthritis, representing a common primary inflammatory arthritis in adult men. Gout is caused by an accumulation of uric acid, precipitating in synovial joints, resulting in a dramatic

\section{Key points}

Low grade fever is common in chronic inflammatory diseases.

In both acute and chronic rheumatic diseases, the inflammatory process is driven by inflammatory mediators that also serve to function as pyrogens.

Drugs commonly used to treat immune-mediated inflammatory disease, such as NSAIDs and corticosteroids, may mask the febrile component of rheumatic disease. Anti-rheumatic drugs may also cause fever.

An acute polyarthritis presenting with fever may well be associated with an acute viral infection (eg parvovirus or arbovirus).

Above all, clinical acumen remains the essential clinical decision-making tool.

KEYWORDS: Inflammatory joint disease, pyrogens, crystals, septic arthritis, vasculitis 
inflammatory response. Systemic fever occurs in a minority of patients with acute gout, reported in around $9 \%$ of cases, but as many as $18 \%$ of people with polyarticular attacks. ${ }^{3}$ Activation of the inflammasome by uric acid crystals, and caspase-dependent processing of pro-interleukin (IL)-1 $\beta$, provide plausible mechanisms for fever in gout. Fever may be more prevalent in calcium pyrophosphate deposition disease (CPPD) than in gout, being reported in as many as $50 \%$ of patients. ${ }^{4}$ There are also numerous reports in the literature of CPPD mimicking systemic disease, with fever as a primary manifestation. ${ }^{5,6}$ However, it is important to bear in mind that CPPD may flare in the context of intercurrent infection and therefore caution must be taken before attributing a febrile episode to CPPD.

\section{Infectious arthritis}

While crystal arthritis and septic arthritis can both result in fever, it is far more common in the latter type. In 1966, Argen reported on 42 cases of septic arthritis, of which only 5 were afebrile. ${ }^{10}$ A more recent prospective study of the clinical features of septic arthritis in Scotland reported lower rates of fever. ${ }^{11}$ Fever was described in only 33 (44\%), and sweats and rigors in only $31 \%$ and $16 \%$ of patients respectively. The fever was less frequent in patients with co-existing rheumatoid arthritis (RA), of whom $43 \%$ were febrile, compared with $70 \%$ of patients without RA. There are a number of likely explanations for these observations, including the suppression of the febrile response by anti-rheumatic drugs, as well as a rising prevalence of prosthetic joints that may associate with more indolent infections.

\section{Polyarthritis and fever}

Presentations with polyarthritis are more likely to be associated with underlying autoimmune disease. By and large, this group of diseases are less frequently associated with fever. Indeed, an acute polyarthritis presenting with fever is more likely to be due to viral infection, such as that seen with parvovirus or arbovirus (eg chikungunya), where fever is typical. ${ }^{12}$

\section{Rheumatoid arthritis}

Fever is not common in RA. While most contemporary publications regarding RA make no mention of fever at all, rheumatologists are familiar with the rare RA patient presenting with aggressive disease characterised by florid polyarticular synovitis accompanied by weight loss and fever. This pattern of disease, where the inflammatory burden is substantial, affects less than $5 \%$ of patients. ${ }^{13}$ When encountered, such manifestations typically prompt extensive investigations for alternative diagnoses, especially malignancy.

\section{Seronegative inflammatory diseases}

In general, the seronegative inflammatory arthritides, including psoriatic arthritis and axial spondyloarthritis (including ankylosing spondyloarthritis), represent conditions with distinct cytokine signatures driving the inflammatory process. It is of interest that these diseases do not respond to blockade of known pyrogens, such as IL-1 and IL-6. Consistent with this, systemic symptoms are generally confined to fatigue and malaise, with fever and weight loss not reported in the published literature. ${ }^{15,16}$

\section{Reactive arthritis}

Reactive arthritis (formerly Reiter's syndrome), the clinical triad of arthritis, urethritis and conjunctivitis, is an aberrant immunological response to recent infection. The two most frequent inciting infections are non-gonococcal urethritis and gastroenteritis. The relationship with fever is not well described. There are a great many published case series of reactive arthritis, and few comment on fever. One large series that systematically assessed fever reported that not one of their 53 patients had experienced high temperature as part of their illness. ${ }^{17}$ Despite the lack of literature base, fever can complicate reactive arthritis, but usually in the very early phase of the disease when the gastrointestinal symptoms are still present. It is therefore worthwhile looking carefully for active infection if fever is detected in a patient with reactive arthritis.

\section{Systemic inflammatory diseases and fever}

\section{Adult-onset Still's disease}

AOSD is the archetypal febrile autoimmune disease. The hallmark of AOSD is fever, which is typically diurnal in nature, rising to a peak often above $40^{\circ} \mathrm{C}$ in the early evening, accompanied by a characteristic salmon-pink rash, with both the fever and rash subsiding completely within hours. ${ }^{18}$ It is often said that a distinguishing feature of the fever in AOSD is the circadian nature of the fever, occurring at the same time each day and completely resolving between episodes, unlike chronic sepsis where the temperature will often fluctuate erratically and may not return to baseline between spikes. In practice, the fever often results in extensive investigation and treatment for infection before a diagnosis of AOSD is reached. ${ }^{19}$ The beneficial effects of blocking IL-6 with tocilizumab suggest that this pyrogen may underpin fever in this syndrome.

\section{Systemic lupus erythematosus}

A hallmark of systemic lupus erythematosus (SLE) is the presence of autoantibodies; immune complexes are potent pyrogens. Fever is well recognised in SLE and may be intermittent, accompanying disease flares or present as a continuous low-grade daily fever. Early descriptions of SLE from the 1950s document fever in active SLE occurring in $86 \%$ of patients. ${ }^{20}$ However, over the ensuing decades the reported incidence of fever attributed to SLE has declined. For example, in patients studied between 1980 and 1989, only 41\% reported fever as a sign of active SLE. ${ }^{21}$ The decline very likely reflects changes in treatment strategies. The titre, as well as the specific patterns of autoantibody positivity correlate to clinical phenotype, and in the context of fever, it has been observed that patients with ribonucleoprotein (RNP) positivity were four times more likely to have fever as a manifestation of their disease. ${ }^{22}$ Given that fever can accompany disease and infection in SLE, an important clinical challenge in SLE is to distinguish 
flare from infection. To this end, C-reactive protein (CRP) levels can be a valuable diagnostic aid, as flares in SLE tend not to cause significant CRP elevations; in the context of SLE, a high CRP has a reported specificity for infection of $84 \% .{ }^{23}$ This is one of the few instances where acute phase reactants can discriminate infection from autoimmunity.

\section{Systemic vasculitis}

It is generally the medium to large vessel vasculitides that are associated with fever. In total, $60 \%$ of patients with polyarteritis nodosa and eosinophilic granulomatosis with polyangiitis (formerly Churg-Strauss syndrome) present with fever. ${ }^{24,25}$ Fever is recognised, but less frequent, in patients with granulomatosis with polyangiitis ((GPA) formerly Wegener's Granulomatosis), with only a quarter of patients having fever documented at presentation. ${ }^{26}$ This lower prevalence of fever in GPA reflects patients presenting with a limited pattern of disease, confined to the orbits and upper respiratory tract. For example, from a detailed review of 13 patients with limited ocular disease, only 5 had evidence of systemic involvement and none had documented fever. ${ }^{27}$

Giant cell arteritis, the most common large vessel vasculitis, presents with fever in a third of patients. ${ }^{28,29}$ Takayasu's arteritis is also an important cause of fever, although the fevers are predominantly noted early on in the disease course..$^{30}$ In a Takayasu's cohort in Mexico, fever was noted in $20 \%$ of patients overall, however fever was present in all the 'prepulseless' patients. ${ }^{31}$ Takayasu's arteritis should be considered an important differential in patients with recurring fever and unexplained elevations in inflammatory markers.

\section{Drugs and fever}

Drugs are an established cause of fever, and in an era of growing levels of polypharmacy, drug fever is a phenomenon that will remain prevalent in years to come. The list of causative drugs is long, and beyond the scope of this review. However, those familiar to the rheumatologist include allopurinol and sulphalasazine, responsible for over $5 \%$ of cases of drug rash with eosinophilia and systemic symptoms (DRESS). ${ }^{33,34}$ Anti-tumour necrosis factor (TNF) therapy, a mainstay of treatment for RA, is associated with a lupus-like syndrome, manifesting as a malar rash and worsening arthralgia, without serious visceral involvement. ${ }^{35}$ Interestingly, in the original report, fever was a prominent feature. $^{36}$

While some drugs may cause fever, a more serious concern to the rheumatologist is the fact that drugs may also mask fever and hide clues to infection that may complicate immune suppression. Aspirin was one of the first anti-rheumatic drugs used, with recognition that it had the ability to both reduce joint inflammation and also fever. ${ }^{37}$ NSAIDs that are widely used as symptomatic treatment for musculoskeletal pain are also effective anti-pyretics, raising the potential for these drugs to mask the febrile component of fever-associated syndromes. Studies exploring the impact of steroids on pyrogen release date back to the 1970 s. ${ }^{38}$ Steroids can mask not only fever, but also many other aspects of systemic infection, with the 'silent abdomen' representing a classic missed diagnosis in acute care.
More recently, the introduction of the biological agent tocilizumab has provided another example of the potential for a drug to suppress an inflammatory response. Tocilizumab is a monoclonal antibody that blocks the IL- 6 pathway that should predictably blunt pyrogenic response. Following on from this, there are a growing number of case reports observing a striking absence of the usual signs of infection (fever and elevated CRP) in patients receiving tocilizumab for RA. ${ }^{40-42}$

\section{Concluding remarks}

The prevalence of fever in different inflammatory diseases is likely to be dictated by the production of specific pyrogens (eg IL-1, IL-6 and immune complexes). Distinct patterns of response to targeted biological therapies in different diseases would seem to support this. A rule of thumb remains that fever must prompt systematic and robust investigation to exclude infection, as the rheumatologist's armamentarium consists of many drugs that may suppress immunity, and to administer these in the context of active infection can be catastrophic.

\section{References}

1 Chadwick J, Mann W. The medical works of Hippocrates. Oxford: Blackwell 1950.

2 Bleeker-Rovers CP, Vos FJ, de Kleijn EM et al. A prospective multicenter study on fever of unknown origin: the yield of a structured diagnostic protocol. Medicine 2007;86:26-38.

3 Lawry GV 2nd, Fan PT, Bluestone R. Polyarticular versus monoarticular gout: a prospective, comparative analysis of clinical features. Medicine 1988;67:335-43.

4 Masuda I, Ishikawa K. Clinical features of pseudogout attack. A survey of 50 cases. Clin Orthop Relat Res 1988:173-81.

5 Berger RG, Levitin PM. Febrile presentation of calcium pyrophosphate dihydrate deposition disease. J Rheumatol 1988;15:642-3.

6 Bong D, Bennett R. Pseudogout mimicking systemic disease. JAMA 1981;246:1438-40.

7 Constantin A, Bouteiller G. Acute neck pain and fever as the first manifestation of chondrocalcinosis with calcification of the transverse ligament of the atlas. Five case-reports with a literature review. Rev Rhum Engl Ed 1998;65:583-5.

8 Draganescu M, Leventhal LJ. Spinal gout: case report and review of the literature. J Clin Rheumatol 2004;10:74-9.

9 Suk KS, Kim KT, Lee SH, Park SW, Park YK. Tophaceous gout of the lumbar spine mimicking pyogenic discitis. Spine J 2007;7:94-9.

10 Argen RJ, Wilson CH Jr, Wood P. Suppurative arthritis. Clinical features of 42 cases. Arch Intern Med 1966;117:661-6.

11 Gupta MN, Sturrock RD, Field M. A prospective 2-year study of 75 patients with adult-onset septic arthritis. Rheumatology 2001;40:24-30.

12 Mourya DT, Mishra AC. Chikungunya fever. Lancet 2006;368: 186-7.

13 Ragan C. The clinical features of rheumatoid arthritis. Prognostic indices. JAMA 1962;181:663-7.

14 Ntatsaki E, Mooney J, Scott DG, Watts RA. Systemic rheumatoid vasculitis in the era of modern immunosuppressive therapy. Rheumatology 2014;53:145-52.

15 Gladman DD, Shuckett R, Russell ML, Thorne JC, Schachter RK. Psoriatic arthritis (PSA) - an analysis of 220 patients. Q J Med 1987;62:127-41.

16 Wilkinson M, Bywaters EG. Clinical features and course of ankylosing spondylitis; as seen in a follow-up of 222 hospital referred cases. Ann Rheum Dis 1958;17:209-28.

17 Sonozaki H, Mitsui H, Miyanaga Y et al. Clinical features of 53 cases with pustulotic arthro-osteitis. Ann Rheum Dis 1981;40:547-53. 
18 McMinn FJ, Bywaters EG. Differences between the fever of Still's disease and that of rheumatic fever. Ann Rheum Dis 1959;18:293-7.

19 Pouchot J, Sampalis JS, Beaudet F et al. Adult Still's disease: manifestations, disease course, and outcome in 62 patients. Medicine 1991;70:118-36.

20 Harvey AM, Shulman LE, Tumulty PA, Conley CL, Schoenrich EH. Systemic lupus erythematosus: review of the literature and clinical analysis of 138 cases. Medicine 1954;33:291-437.

21 Pistiner M, Wallace DJ, Nessim S, Metzger AL, Klinenberg JR. Lupus erythematosus in the 1980s: a survey of 570 patients. Semin Arthritis Rheum 1991;21:55-64.

22 Hoffman IE, Peene I, Meheus L et al. Specific antinuclear antibodies are associated with clinical features in systemic lupus erythematosus. Ann Rheum Dis 2004;63:1155-8.

23 Firooz N, Albert DA, Wallace DJ, Ishimori M, Berel D, Weisman $\mathrm{MH}$. High-sensitivity C-reactive protein and erythrocyte sedimentation rate in systemic lupus erythematosus. Lupus 2011;20:588-97.

24 Pagnoux C, Seror R, Henegar C et al. Clinical features and outcomes in 348 patients with polyarteritis nodosa: a systematic retrospective study of patients diagnosed between 1963 and 2005 and entered into the French Vasculitis Study Group Database. Arthritis Rheum 2010;62:616-26.

25 Guillevin L, Cohen P, Gayraud M, Lhote F, Jarrousse B, Casassus P. Churg-Strauss syndrome. Clinical study and long-term follow-up of 96 patients. Medicine 1999;78:26-37.

26 Hoffman GS, Kerr GS, Leavitt RY et al. Wegener granulomatosis: an analysis of 158 patients. Ann Intern Med 1992;116:488-98.

27 Perry SR, Rootman J, White VA. The clinical and pathologic constellation of Wegener granulomatosis of the orbit. Ophthalmology 1997;104:683-94.

28 Myklebust G, Gran JT. A prospective study of 287 patients with polymyalgia rheumatica and temporal arteritis: clinical and laboratory manifestations at onset of disease and at the time of diagnosis. Br J Rheumatol 1996;35:1161-8.

29 Machado EB, Michet CJ, Ballard DJ et al. Trends in incidence and clinical presentation of temporal arteritis in Olmsted County, Minnesota, 1950-1985. Arthritis Rheum 1988;31:745-9.

30 Hall S, Barr W, Lie JT, Stanson AW, Kazmier FJ, Hunder GG. Takayasu arteritis. A study of 32 North American patients. Medicine 1985;64:89-99.
31 Soto ME, Espinola N, Flores-Suarez LF, Reyes PA. Takayasu arteritis: clinical features in 110 Mexican Mestizo patients and cardiovascular impact on survival and prognosis. Clin Exp Rheumatol 2008;26:S9-15.

32 Martinez-Taboada VM, Blanco R, Garcia-Fuentes M, RodriguezValverde V. Clinical features and outcome of 95 patients with hypersensitivity vasculitis. Am J Med 1997;102:186-91.

33 Um SJ, Lee SK, Kim YH et al. Clinical features of drug-induced hypersensitivity syndrome in 38 patients. J Investig Allergol Clin Immunol 2010;20:556-62.

34 Ramasamy SN, Korb-Wells CS, Kannangara DR et al. Allopurinol hypersensitivity: a systematic review of all published cases, 19502012. Drug Saf 2013;36:953-80.

35 Williams EL, Gadola S, Edwards CJ. Anti-TNF-induced lupus. Rheumatology 2009;48:716-20.

36 Shakoor N, Michalska M, Harris CA, Block JA. Drug-induced systemic lupus erythematosus associated with etanercept therapy. Lancet 2002;359:579-80.

37 Maclagan T. The treatment of rheumatism by salicin and salicylic acid. Br Med J 1876;1:627.

38 Dillard GM, Bodel P. Studies on steroid fever. II. Pyrogenic and anti-pyrogenic activity in vitro of some endogenous steroids of man. J Clin Invest 1970;49:2418-26.

39 Picard C, von Bernuth H, Ghandil P et al. Clinical features and outcome of patients with IRAK-4 and MyD88 deficiency. Medicine 2010;89:403-25.

40 Yanagawa Y, Hirano Y, Kato H, Iba T. The absence of typical pneumonia symptoms in a patient with rheumatoid arthritis during tocilizumab and steroid treatment. BMJ Case Rep 2012; pii:bcr0220125835.

41 Bari SF, Khan A, Lawson T. C reactive protein may not be reliable as a marker of severe bacterial infection in patients receiving tocilizumab. BMJ Case Rep 2013;pii:bcr2013010423.

42 Fujiwara H, Nishimoto N, Hamano Y et al. Masked early symptoms of pneumonia in patients with rheumatoid arthritis during tocilizumab treatment: a report of two cases. Mod Rheumatol 2009;19:64-8.

Address for correspondence: Dr J Galloway, Academic Department of Rheumatology, 10 Cutcombe Road, King's College London, London SE5 9RJ, UK.

Email: james.galloway@nhs.net 